\title{
On a Hibernation Plankton-Nutrient Chemostat Model with Delayed Response in Growth
}

\author{
Juan Ma, Mehbuba Rehim \\ College of Mathematics and System Sciences, Xinjiang University, Urumqi, China \\ Email: 1365417352@qq.com
}

How to cite this paper: Ma, J. and Rehim, M. (2017) On a Hibernation PlanktonNutrient Chemostat Model with Delayed Response in Growth. Journal of Applied Mathematics and Physics, 5, 45-58. http://dx.doi.org/10.4236/jamp.2017.51007

Received: October 16, 2016

Accepted: January 1, 2017

Published: January 4, 2017

Copyright $\odot 2017$ by authors and Scientific Research Publishing Inc. This work is licensed under the Creative Commons Attribution International License (CC BY 4.0).

http://creativecommons.org/licenses/by/4.0/

\begin{abstract}
In this paper, a hibernation plankton-nutrient chemostat model with delayed response in growth is considered. By using the stroboscopic map and the theorem of impulsive delay differential equation, a plankton-extinction boundary periodic solution is obtained. The sufficient conditions on the permanence and globally attractive of the chemostat system are also obtained. Our main results reveal that the delayed response in growth plays an important role on the dynamical behaviors of system.
\end{abstract}

\section{Keywords}

Chemostat Model, Hibernation, Delayed Response in Growth, Permanence, Extinction

\section{Introduction}

The chemostat is an important experimental instrument used to provide a controlled environment. Under this condition, the experimenter can adjust the parameters of system and get the final outcome. This chemostat model had been discussed by Smith and Waltman in [1]. In fact, the taken nutrient will not immediately absorbed by microorganism. In other words, nutrients with transformation from the substrate to microorganism have a lag time. Many scholars [2] [3] [4] [5] make discussion about chemostat model with discrete time delay. However, the system will have some changes because of the influence of climate; these perturbations break the continuity of the system. So the impulsive differential equations are considered into the system in [6] [7] [8] [9]. It is important for us to know more about ecology.

In recent years, some authors pay more attention to the hibernation of the plankton. The hibernation has an important sense of adaptation in ecology. Due to unfavorable environmental conditions, the plankton enters a hibernation state 
in advance. In order to save energy, plankton must maintain the weak life period overcoming the difficulties, such as drought stress, cold climate and temperature. The pressures elimination will restore growth. By hibernation, animals can reduce energy requirement and survive a few months in [10]. Some scholars also proposed that hibernation can make animals through hardship on cold environments and limited availability of food in [11]. However, there are many factors of plankton movements in the lakes, such as currents and river diffusion. These researches are seen in Levin and Segel [12] and Okubo [13]. Ruan discussed Turing instability and the existence of travelling wave solutions in [14].

Furthermore, it is necessary to study a chemostat model with hibernation and impulsive diffusion on nutrients. In [15], the author considered the dynamics of a plankton-nutrient chemostat model with hibernation and it was described by impulsive switched systems as follows

$$
\left\{\begin{array}{r}
\dot{S}_{1}(t)=D\left(S_{1}^{0}-S_{1}(t)\right), \\
\dot{S}_{2}(t)=-D S_{2}(t)-\frac{m S_{2}(t) x(t)}{K_{1}\left(K+S_{2}(t)\right)}, \\
\dot{x}(t)=-D x(t)+\frac{m S_{2}(t) x(t)}{K+S_{2}(t)}, \\
S_{1}\left(t^{+}\right)=S_{1}(t)-d S_{1}(t), \\
S_{2}\left(t^{+}\right)=S_{2}(t)+d S_{1}(t), \\
x\left(t^{+}\right)=x(t), \\
\dot{S}_{1}(t)=D\left(S_{1}^{0}-S_{1}(t)\right), \\
\dot{S}_{2}(t)=-D S_{2}(t), \\
\dot{x}(t)=-d_{1} x(t), \\
S_{1}\left(t^{+}\right)=S_{1}(t)+\mu_{1}, \\
S_{2}\left(t^{+}\right)=S_{2}(t)+\mu_{2}, \\
x\left(t^{+}\right)=x(t) .
\end{array}\right\} t=((n+L) \tau,
$$

where $\tau \in R_{+}=[0, \infty), n \in Z_{+}, Z_{+}$is the set of all positive integers; $S_{1}(t)$ and $S_{2}(t)$ represent the concentration of the nutrient in the river and reservoir at time $t$ respectively. $x(t)$ is the concentration of the plankton in the reservoir at time $t . S_{1}^{0}$ is the input nutrient concentration in the river. $D$ is the dilution rate. $K_{1}$ is the yield of plankton $x(t)$ per unit mass of substrate. $d_{1}$ is the death rate of the plankton in the intervals of hibernation. $S_{1}\left((n+L) \tau^{+}\right)$and $S_{2}\left((n+L) \tau^{+}\right)$are the concentration of the nutrient in the river and reservoir immediately after the $n$th diffusion pulse at time $t=n \tau$ respectively, while $S_{1}((n+L) \tau)$ and $S_{2}((n+L) \tau)$ are the concentration of the nutrient in the river and reservoir before the $n$th diffusion pulse at time $t=(n+L) \tau$ separately. Due to the effect climate, the period of system is divided into two sections. That is normal seasons and drought seasons. In the normal seasons, the plank- 
ton grow regularly. The plankton is in hibernation in the drought seasons. $t=(n+L) \tau$ are moments of torrential rain, the nutrient is diffusing between rivers and reservoir in moments of torrential rain. $t=(n+1) \tau$ are moments of rainy season. $\mu_{1}$ and $\mu_{2}$ are the amount of nutrients coming from surrounding soil in moments of rainy season.

Based on the above discussion, we consider the following a hibernation plankton-nutrient chemostat model with delayed response in growth

$$
\begin{aligned}
& \dot{S}_{1}(t)=D\left(S_{0}-S_{1}(t)\right), \\
& \left.\dot{S}_{2}(t)=-D S_{2}(t)-\frac{m S_{2}(t) x(t)}{\delta\left(K+S_{2}(t)\right)},\right\} t \in(n \tau,(n+L) \tau], \\
& \dot{x}(t)=-D x(t)+\mathrm{e}^{-D \tau_{1}} \frac{m S_{2}\left(t-\tau_{1}\right) x\left(t-\tau_{1}\right)}{K+S_{2}\left(t-\tau_{1}\right)}, \\
& S_{1}\left(t^{+}\right)=(1-d) S_{1}(t) \\
& \left.S_{2}\left(t^{+}\right)=S_{2}(t)+d S_{1}(t),\right\} t=(n+L) \tau \text {, } \\
& x\left(t^{+}\right)=x(t) \\
& \dot{S}_{1}(t)=D\left(S_{0}-S_{1}(t)\right) \\
& \left.\dot{S}_{2}(t)=-D S_{2}(t),\right\} t \in((n+L) \tau,(n+1) \tau], \\
& \dot{x}(t)=-d_{1} x(t), \\
& S_{1}\left(t^{+}\right)=S_{1}(t)+\mu_{1} \text {, } \\
& \left.S_{2}\left(t^{+}\right)=S_{2}(t)+\mu_{2},\right\} t=(n+1) \tau \text {. } \\
& x\left(t^{+}\right)=x(t) .
\end{aligned}
$$

Suppose system (1.2) is connected by impulsive diffusion spread between rivers and reservoirs. There is no nutrients input in reservoir. Nutrient input is thought to come from the upper stream. Where constant $\tau_{1} \geq 0$ represents the time delay involved in the conversion of nutrient to plankton. Due to the chemostat outflow, $\mathrm{e}^{-D \tau_{1}}$ is the positive constant, since it is assumed that the current change in biomass depends on the amount of nutrient consumed $\tau_{1}$ units of time before time $t$ and that survive in the chemostat the $\tau_{1}$ units of time assumed necessary to complete the nutrient conversion process. Other parameters are the same as system (1.1). $x(t)$ is continuous on $(n \tau,(n+L) \tau]$ and $((n+L) \tau,(n+1) \tau]$, there exists $x\left((n+L) \tau^{+}\right)=\lim _{t \rightarrow\left[(n+L) \tau^{+}\right]} x(t)$ and $x\left((n+1) \tau^{+}\right)=\lim _{t \rightarrow\left[(n+1) \tau^{+}\right]} x(t)$.

For system (1.2), we will discuss the sufficient and necessary conditions for the permanence and extinction. This paper can be summarized as follows. In Section 2, we present some preliminary results about system (1.2). Our results about extinction are stated and proven in Section 3. In Section 4, we study the permanence of system (1.2). Finally, we give a brief discussion and numerical analysis. 


\section{Preliminary Results}

In this part, we will give some lemmas which will be useful for our main results.

Lemma 1. [16] Consider the following impulsive differential system

$$
\begin{cases}\dot{w}(t) \leq(\geq) p(t) w(t)+q(t), & t \neq t_{k}, k \in N \\ w\left(t_{k}^{+}\right) \leq(\geq) d_{k} w\left(t_{k}\right)+b_{k}, & t=t_{k}, k \in N .\end{cases}
$$

where $p(t), q(t) \in c\left(R_{+}, R\right), d_{k} \geq 0$, and $b_{k}$ is the constant. Assume $\left(A_{1}\right)$ the sequence $t_{k}$ satisfies $0 \leq t_{0}<t_{1}<t_{2}<\cdots$, with $\lim _{t \rightarrow \infty} t_{k}^{+}=\infty ;\left(A_{2}\right)$ $w \in P C^{\prime}\left(R_{+}, R\right)$ and $\mathrm{w}(\mathrm{t})$ is left-continuous at $t_{k}, k \in N$. Then

$$
\begin{aligned}
w(t) \leq(\geq) \quad w\left(t_{0}\right) \prod_{t_{0}<t_{k}<t} d_{k} \exp \left(\int_{t_{0}}^{t} p(s) \mathrm{d} s\right) & +\sum_{t_{0}<t_{k}<t}\left(\prod_{t_{k}<t_{j}<t} d_{j} \exp \left(\int_{t_{k}}^{t} p(s) \mathrm{d} s\right)\right) b_{k} \\
& +\int_{t_{0}}^{t} \prod_{s<t_{k}<t} d_{k} \exp \left(\int_{s}^{t} p(\theta) \mathrm{d} \theta\right) q(s) \mathrm{d} s, t \geq t_{0} .
\end{aligned}
$$

Lemma 2. [17] Consider the following delay differential equation:

$$
\dot{x}(t)=r_{1} x\left(t-\tau_{1}\right)-r_{2} x(t),
$$

where $a, b$ and $\tau_{1}$ are all positive constants and $x(t)>0$ for $t \in\left[-\tau_{1}, 0\right]$.

a) If $r_{1}<r_{2}$, then $\lim _{t \rightarrow \infty} x(t)=0$;

b) If $r_{1}>r_{2}$, then $\lim _{t \rightarrow \infty} x(t)=\infty$.

Lemma 3. For any positive solution $\left(S_{1}(t), S_{2}(t), x(t)\right)$ of system (1.2) satisfy $S_{1}(0)>, S_{2}(0)>0, x(0)>0$, there exists a constant $M>0$, such that

$$
\limsup _{t \rightarrow \infty} S_{1}(t) \leq M, \quad \limsup _{t \rightarrow \infty} S_{2}(t) \leq M, \quad \limsup x(t) \leq M \text {, }
$$

where $M=\frac{D S_{0}}{\lambda}+\frac{\left(\mu_{1}+\mu_{2}\right) \mathrm{e}^{\lambda \tau}}{\mathrm{e}^{\lambda \tau}-1}, \lambda=\min \left\{D, d_{1}\right\}$.

The proof of Lemma 3 is simple so we omit it here.

\section{Extinction}

The solution of system (1.2) corresponding to $x(t)=0$ is called plankton-extinction periodic solution. For system (1.2), if we select $x(t) \equiv 0$, then system (1.2) becomes the following model

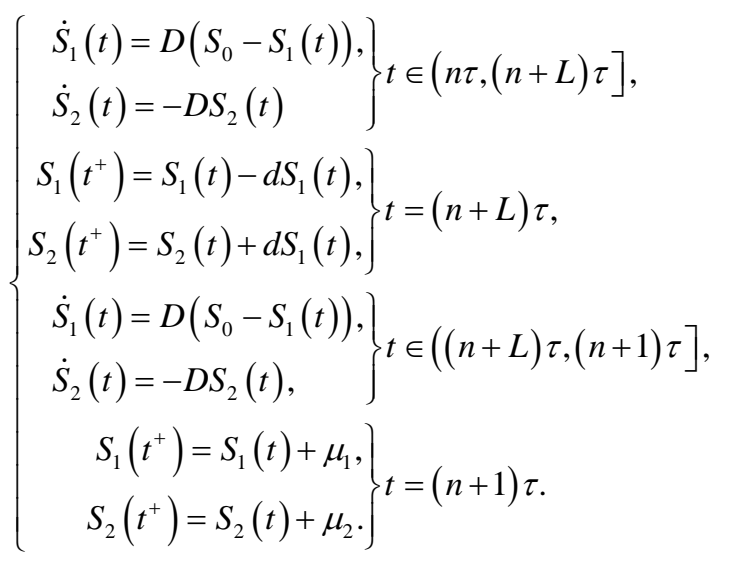

Integrating and solving the system (3.1) equations between pulses, we have 


$$
\left\{\begin{array}{l}
S_{1}(t)=\left\{\begin{array}{l}
S_{0}-\left(S_{0}-S_{1}\left(n \tau^{+}\right)\right) \mathrm{e}^{-D(t-n \tau)}, n \tau<t \leq(n+L) \tau, \\
S_{0}-\left[S_{0}-(1-d)\left(S_{0}-\left(S_{0}-S_{1}\left(n \tau^{+}\right)\right) \mathrm{e}^{-D L \tau}\right)\right] \mathrm{e}^{-D(t-(n+L) \tau)}, \quad(n+L) \tau<t \leq(n+1) \tau,
\end{array}\right. \\
S_{2}(t)=\left\{\begin{array}{l}
S_{2}\left(n \tau^{+}\right) \mathrm{e}^{-D(t-n \tau)}, n \tau<t \leq(n+L) \tau, \\
{\left[\begin{array}{l}
\left.S_{2}\left(n \tau^{+}\right) \mathrm{e}^{-D L \tau}+d\left(S_{0}-\left(S_{0}-S_{1}\left(n \tau^{+}\right)\right) \mathrm{e}^{-D L \tau}\right)\right] \mathrm{e}^{-D(t-(n+L) \tau)},(n+L) \tau<t \leq(n+1) \tau .
\end{array}\right.}
\end{array}\right.
\end{array}\right.
$$

Consider the stroboscopic map of system (3.1), from the third, fourth, seventh and eighth equations of system (3.1) we have:

$$
\begin{cases}S_{1}\left((n+1) \tau^{+}\right)=(1-d) \mathrm{e}^{-D \tau} S_{1}\left(n \tau^{+}\right)+A, & n \tau<t \leq(n+L) \tau, \\ S_{2}\left((n+1) \tau^{+}\right)=d \mathrm{e}^{-D \tau} S_{1}\left(n \tau^{+}\right)+\mathrm{e}^{-D \tau} S_{2}\left(n \tau^{+}\right)+B, & n \tau<t \leq(n+L) \tau .\end{cases}
$$

where $A=\left[1-\mathrm{e}^{-D(1-L) \tau}+(1-d) \mathrm{e}^{-D(1-L) \tau}-(1-d) \mathrm{e}^{(-D \tau)}\right] S_{0}+\mu_{1}$, $B=d S_{0}\left(\mathrm{e}^{-D(1-L) \tau}-\mathrm{e}^{-D \tau}\right)+\mu_{2}$. Equation (3.3) is difference equations. The dynamical behaviors of system (3.3) with equation (3.2) have been decided to the dynamical behaviors of system (3.1). So we focus on discussing System (3.3). System (3.3) has the following unique solution

$$
\left\{\begin{array}{l}
S_{1}^{*}=\frac{A}{1-(1-d) \mathrm{e}^{-D \tau}}>0, \\
S_{2}^{*}=\frac{B\left[1-(1-d) \mathrm{e}^{-D \tau}\right]+A d \mathrm{e}^{-D \tau}}{\left(1-\mathrm{e}^{-D \tau}\right)\left[1-(1-d) \mathrm{e}^{-D \tau}\right]}>0 .
\end{array}\right.
$$

To change System (3.3) to a map, we define the map $F: R_{+}^{2} \rightarrow R_{+}^{2}$ :

$$
\left\{\begin{array}{l}
F_{1}(x)=(1-d) \mathrm{e}^{-D \tau} x_{1}+A, \\
F_{2}(x)=d \mathrm{e}^{-D \tau} x_{1}+\mathrm{e}^{-D \tau} x_{2}+B .
\end{array}\right.
$$

$F(x)$ is the map calculate at the point $x=\left(x_{1}, x_{2}\right) \in R_{+}^{2}$. According to the lemma 3.2 and 3.4 of Refer [15], we obtain

$$
\left\{\begin{array}{l}
\widetilde{S_{1}(t)}=\left\{\begin{array}{l}
S_{0}-\left(S_{0}-S_{1}^{*}\right) \mathrm{e}^{-D(t-n \tau)}, n \tau<t \leq(n+L) \tau, \\
S_{0}-\left[S_{0}-(1-d)\left(S_{0}-\left(S_{0}-S_{1}^{*}\right) \mathrm{e}^{-D L \tau}\right)\right] \mathrm{e}^{-D(t-(n+L) \tau)},(n+L) \tau<t \leq(n+1) \tau,
\end{array}\right. \\
\widetilde{S_{2}(t)}=\left\{\begin{array}{l}
S_{2}^{*} \mathrm{e}^{-D(t-n \tau)}, n \tau<t \leq(n+L) \tau, \\
{\left[\begin{array}{l}
\left.S_{2}^{*} \mathrm{e}^{-D L \tau}+d\left(S_{0}-\left(S_{0}-S_{1}^{*}\right) \mathrm{e}^{-D L \tau}\right)\right] \mathrm{e}^{-D(t-(n+L) \tau)},(n+L) \tau<t \leq(n+1) \tau .
\end{array}\right.}
\end{array}\right.
\end{array}\right.
$$

Hence, system (1.2) has a positive plankton-extinction periodic solution $\left(\widetilde{S_{1}(t)}, \widetilde{S_{2}(t)}, 0\right)$. In what follows, we will study the globally attractive of the plankton-extinction periodic solution $\left(\widehat{S_{1}(t)}, \widehat{S_{2}(t)}, 0\right)$ of system (1.2).

Theorem 1 . The periodic solution $\left(\widetilde{S_{1}(t)}, \widetilde{S_{2}(t)}, 0\right)$ of system $(1.2)$ is globally attractive, if

$$
\mathrm{e}^{-D \tau_{1}} \frac{m \hat{S}_{2}}{K+\hat{S}_{2}}<D
$$


where $\hat{S}_{2}=S_{2}^{*}\left(\mathrm{e}^{-D \tau}+\mathrm{e}^{-D L \tau}\right)+d S_{1}^{*} \mathrm{e}^{-D \tau}+d S_{0} \mathrm{e}^{-D \tau}\left(\mathrm{e}^{D L \tau}-1\right)$ and parametric $S_{1}^{*}$ and $S_{2}^{*}$ are given in (3.4).

Proof. Suppose $\left(S_{1}(t), S_{2}(t), x(t)\right)$ is any positive solution of system (1.2) with $S_{1}(0)>0, S_{2}(0)>0, x(0)>0$. Based on the condition (3.7), we set $P(z)=\frac{m \mathrm{e}^{-D \tau_{1}} \mathrm{z}}{K+z}$, then $P(z)$ is strictly increasing function for arbitrary $z \geq 0$. We may select sufficiently small $\varepsilon>0$, such that

$$
\frac{m \mathrm{e}^{-D \tau_{1}} \eta}{K+\eta}<D,
$$

where $\eta=\hat{S}_{2}+\varepsilon$. From the second equation of (1.2) we have $\dot{S}(t) \leq-D S(t)$.

Consider the following equations with pulse

$$
\left\{\begin{array}{c}
\dot{Z}_{1}(t)=D\left(S_{0}-Z_{1}(t)\right) \\
\dot{Z}_{2}(t)=-D Z_{2}(t) \\
Z_{1}\left(t^{+}\right)=Z_{1}(t)-d Z_{1}(t), \\
Z_{2}\left(t^{+}\right)=Z_{2}(t)+d Z_{1}(t), \\
\dot{Z}_{1}(t)=D\left(S_{0}-Z_{1}(t)\right), \\
\dot{Z}_{2}(t)=-D Z_{2}(t), \\
Z_{1}\left(t^{+}\right)=Z_{1}(t)+\mu_{1}, \\
Z_{2}\left(t^{+}\right)=Z_{2}(t)+\mu_{2} \cdot
\end{array}\right\} t=(n+L) \tau,
$$

From(3.6) and (3.9) we have that $\widetilde{S_{1}(t)} \leq Z_{1}(t), \widetilde{S_{2}(t)} \leq Z_{2}(t)$ and $Z_{1}(t) \rightarrow \widehat{S_{1}(t)}, Z_{2}(t) \rightarrow \widehat{S_{2}(t)}$ as $t \rightarrow \infty$. Therefore, there exist a integer $n_{1}$ and an arbitrary positive parameter $\varepsilon$, such that

$$
S_{2}(t) \leq \hat{S}_{2}+\varepsilon=: \eta
$$

for all $t \geq n_{1} \tau$, where $\hat{S}_{2}=S_{2}^{*}\left(\mathrm{e}^{-D \tau}+\mathrm{e}^{-D L \tau}\right)+d S_{1}^{*} \mathrm{e}^{-D \tau}+d S_{0} \mathrm{e}^{-D \tau}\left(\mathrm{e}^{D L \tau}-1\right)$. For $t>n_{1} \tau+\tau_{1}$, from (3.10) and the second equation of (1.1), we have

$$
\dot{x}(t) \leq \frac{m \mathrm{e}^{-D \tau_{1}} \eta}{K+\eta} x\left(t-\tau_{1}\right)-D x(t) .
$$

Consider the following impulsive differential equation

$$
\dot{y}(t)=\frac{m \mathrm{e}^{-D \tau_{1}} \eta}{K+\eta} y\left(t-\tau_{1}\right)-D y(t) .
$$

According to lemma 2 and condition (3.8), we obtain that $\lim _{t \rightarrow \infty} y(t)=0$. Since $X(s)=y(s)>0$ when $s \in\left[-\tau_{1}, 0\right]$, by the impulsive delay differential equation and the nonnegative of the solutions, we obtain $x(t) \rightarrow 0$ as $t \rightarrow \infty$. Without loss of generality, for all $t \geq 0$, we may suppose that $0<x(t)<\varepsilon$. By the second equation of the system (1.2), we have

$$
\dot{S}_{2}(t) \geq-\left(D+\frac{m \varepsilon}{\delta K}\right) S_{2}(t)
$$

Consider the following comparison system with pulse 


$$
\begin{aligned}
& \left\{\begin{array}{l}
\dot{Z}_{3}(t)=D\left(S_{0}-Z_{3}(t)\right), \\
\dot{Z}_{4}(t)=-\left(D+\frac{m \varepsilon}{\delta K}\right) Z_{4}(t)
\end{array}\right\} t \in(n \tau,(n+L) \tau], \\
& \left\{\begin{array}{l}
Z_{3}\left(t^{+}\right)=Z_{3}(t)-d Z_{3}(t), \\
Z_{4}\left(t^{+}\right)=Z_{4}(t)+d Z_{3}(t),
\end{array}\right\} t=(n+L) \tau, \\
& \left.\begin{array}{l}
\dot{Z}_{3}(t)=D\left(S_{0}-Z_{3}(t)\right), \\
\dot{Z}_{4}(t)=-D Z_{4}(t),
\end{array}\right\} t \in((n+L) \tau,(n+1) \tau], \\
& \left.\begin{array}{l}
Z_{3}\left(t^{+}\right)=Z_{3}(t)+\mu_{1}, \\
Z_{4}\left(t^{+}\right)=Z_{4}(t)+\mu_{2} \cdot
\end{array}\right\} t=(n+1) \tau .
\end{aligned}
$$

The system (3.11) has a positive solution $\left(\overline{Z_{3}(t)}, \overline{Z_{4}(t)}\right)$, where $\left(\widetilde{Z_{3}(t)}, \overline{Z_{4}(t)}\right)$ are expressed as follows

$$
\left\{\begin{array}{l}
\widetilde{Z_{3}(t)}=\left\{\begin{array}{l}
S_{0}-\left(S_{0}-S_{1}^{*}\right) \mathrm{e}^{-D(t-n \tau)}, n \tau<t \leq(n+L) \tau, \\
S_{0}-\left[S_{0}-(1-d)\left(S_{0}-\left(S_{0}-S_{1}^{*}\right) \mathrm{e}^{-D L \tau}\right)\right] \mathrm{e}^{-D(t-(n+L) \tau)},(n+L) \tau<t \leq(n+1) \tau,
\end{array}\right. \\
\widetilde{Z_{4}(t)}=\left\{\begin{array}{l}
Z_{4}^{*} \mathrm{e}^{-\left(D+\frac{m \varepsilon}{\delta K}\right)(t-n \tau)}, n \tau<t \leq(n+L) \tau, \\
{\left[Z_{4}^{*} \mathrm{e}^{-\left(D+\frac{m \varepsilon}{\delta K}\right) L \tau}+d\left(S_{0}-\left(S_{0}-S_{1}^{*}\right) \mathrm{e}^{-D L \tau}\right)\right] \mathrm{e}^{-D(t-(n+L) \tau)},(n+L) \tau<t \leq(n+1) \tau .}
\end{array}\right.
\end{array}\right.
$$

In which

$$
Z_{4}^{*}=\frac{B\left[1-(1-d) \mathrm{e}^{-D \tau}\right]+A d \mathrm{e}^{-D \tau}}{\left(1-\mathrm{e}^{-\left(D+\frac{m \varepsilon L}{\delta K}\right) \tau}\right)\left[1-(1-d) \mathrm{e}^{-D \tau}\right]}>0
$$

and $A=\left[1-\mathrm{e}^{-D(1-L) \tau}+(1-d) \mathrm{e}^{-D(1-L) \tau}-(1-d) \mathrm{e}^{(-D \tau)}\right] S_{0}+\mu_{1}$,

$B=d S_{0}\left(\mathrm{e}^{-D(1-L) \tau}-\mathrm{e}^{-D \tau}\right)+\mu_{2}$. For arbitrary $\varepsilon>0$, there exists a constant $T_{1}>0$, such that, for all $T>T_{1}$,

$$
\widetilde{Z_{3}(t)}-\varepsilon_{1}<S_{1}(t)<\widetilde{S_{1}(t)}+\varepsilon_{1}, \widetilde{Z_{4}(t)}-\varepsilon_{1}<S_{2}(t)<\widetilde{S_{2}(t)}+\varepsilon_{1} .
$$

Let $\varepsilon \rightarrow 0$, we obtain

$$
\widetilde{S_{1}(t)}-\varepsilon_{1}<S_{1}(t)<\widetilde{S_{1}(t)}+\varepsilon_{1}, \widetilde{S_{2}(t)}-\varepsilon_{1}<S_{2}(t)<\widetilde{S_{2}(t)}+\varepsilon_{1}
$$

For $t \rightarrow \infty$ and $\varepsilon_{1} \rightarrow 0, S_{1}(t) \rightarrow \widetilde{S_{1}(t)}$ and $S_{2}(t) \rightarrow \widetilde{S_{2}(t)}$. The proof of Theorem 1 is completed.

\section{Permanence}

In this section we shall study the permanence of system (1.2).

Theorem 2. System (1.2) is permanent, if

$$
\mathrm{e}^{-D \tau_{1}} \frac{m \breve{S}_{2}}{K+\breve{S}_{2}}>D .
$$


where $\breve{S}_{2}=Z_{6}^{*}\left(\mathrm{e}^{-\left(D+\frac{m m_{1}^{*}}{\delta K}\right) L \tau}+\mathrm{e}^{-\left(D+\frac{m m_{1}^{*} L}{\delta K}\right) \tau}\right)+d S_{1}^{*} \mathrm{e}^{-D \tau}+d S_{0} \mathrm{e}^{-D \tau}\left(\mathrm{e}^{D L \tau}+1\right), \quad S_{1}^{*}$ and $Z_{6}^{*}$ are given in (3.4) and (4.8) respectively.

Proof. Suppose $\left(S_{1}(t), S_{2}(t), x(t)\right)$ is any positive solution of system (1.2) with $S_{1}(0)>0, S_{2}(0)>0, x(0)>0$. We may rewrite the second equation of the system (1.2) as follows

$$
\dot{x}(t)=\left(\frac{m \mathrm{e}^{-D \tau_{1}} S_{2}(t)}{K+S_{2}(t)}-D\right) x(t)-m \mathrm{e}^{-D \tau_{1}} \frac{\mathrm{d}}{\mathrm{d} t} \int_{t-\tau_{1}}^{t} \frac{S_{2}(\theta) X(\theta)}{K+S_{2}(\theta)} \mathrm{d} \theta .
$$

Define

$$
V(t)=x(t)+m \mathrm{e}^{-D \tau_{1}} \int_{t-\tau_{1}}^{t} \frac{S_{2}(\theta) X(\theta)}{K+S_{2}(\theta)} \mathrm{d} \theta
$$

Derive $V(t)$ along with solution of system (1.2), we obtain

$$
\dot{V}(t)=D\left[\frac{m \mathrm{e}^{-D \tau_{1}} S_{2}(t)}{D\left(k+S_{2}(t)\right)}-1\right] x(t) .
$$

Set

$$
m_{1}^{*}=\frac{\delta K}{m}\left[\frac{1}{\tau} \ln \frac{s_{2}^{*}\left(m e^{-D \tau_{1}}-D\right)}{K}-D\right] .
$$

From (4.4) we obtain $m_{1}^{*}>0$. We may select a positive integer $\varepsilon_{1}$ small enough, such that

$$
\frac{m \mathrm{e}^{-D \tau_{1}} \beta}{D(K+\beta)}>1
$$

where $\beta=Z_{6}^{*}\left(\mathrm{e}^{-\left(D+\frac{m m_{1}^{*}}{\delta K}\right) L \tau}+\mathrm{e}^{-\left(D+\frac{m m_{L}^{*} L}{\delta K}\right) \tau}\right)+d S_{1}^{*} \mathrm{e}^{-D \tau}+d S_{0} \mathrm{e}^{-D \tau}\left(\mathrm{e}^{D L \tau}+1\right)-\varepsilon_{1}$. For any nonnegative integer $t_{0}$, we claim that inequality $x(t)<m_{1}^{*}$ is not hold for all $t \geq t_{0}$. Otherwise, there exists a positive parameter $T_{1}$, such that $x(t)<m_{1}^{*}$ for all $t>T_{1}$. From the system (1.2) we obtain

$$
\begin{aligned}
& \left(\begin{array}{l}
\dot{S}_{1}(t)=D\left(S_{0}-S_{1}(t)\right), \\
\dot{S}_{2}(t) \geq-\left(D+\frac{m m_{1}^{*}}{\delta K}\right) S_{2}(t)
\end{array}\right\} t \in(n \tau,(n+L) \tau], \\
& \left.\begin{array}{l}
S_{1}\left(t^{+}\right)=S_{1}(t)-d S_{1}(t), \\
S_{2}\left(t^{+}\right)=S_{2}(t)+d S_{1}(t),
\end{array}\right\} t=(n+L) \tau, \\
& \left.\begin{array}{l}
\dot{S}_{1}(t)=D\left(S_{0}-S_{1}(t)\right), \\
\dot{S}_{2}(t)=-D S_{2}(t),
\end{array}\right\} t \in((n+L) \tau,(n+1) \tau], \\
& \left.\begin{array}{c}
S_{1}\left(t^{+}\right)=S_{1}(t)+\mu_{1}, \\
S_{2}\left(t^{+}\right)=S_{2}(t)+\mu_{2} .
\end{array}\right\} t=(n+1) \tau .
\end{aligned}
$$


Consider the following impulsive differential equation

$$
\begin{aligned}
& \left\{\begin{array}{l}
\dot{Z}_{5}(t)=D\left(S_{0}-Z_{5}(t)\right), \\
\dot{Z}_{6}(t)=-\left(D+\frac{m m_{1}^{*}}{\delta K}\right) Z_{6}(t)
\end{array}\right\} t \in(n \tau,(n+L) \tau], \\
& \left.\begin{array}{l}
Z_{5}\left(t^{+}\right)=Z_{5}(t)-d Z_{5}(t), \\
Z_{6}\left(t^{+}\right)=Z_{6}(t)+d Z_{5}(t),
\end{array}\right\} t=(n+L) \tau, \\
& \left.\begin{array}{l}
\dot{Z}_{5}(t)=D\left(S_{0}-Z_{5}(t)\right), \\
\dot{Z}_{6}(t)=-D Z_{6}(t),
\end{array}\right\} t \in((n+L) \tau,(n+1) \tau], \\
& \left.\begin{array}{l}
Z_{5}\left(t^{+}\right)=Z_{5}(t)+\mu_{1}, \\
Z_{6}\left(t^{+}\right)=Z_{6}(t)+\mu_{2} \cdot
\end{array}\right\} t=(n+1) \tau .
\end{aligned}
$$

The system (4.6) has a unique globally asymptotically stable positive solution as follows

$$
\left\{\begin{array}{l}
\widetilde{Z_{5}(t)}=\left\{\begin{array}{l}
S_{0}-\left(S_{0}-S_{1}^{*}\right) \mathrm{e}^{-D(t-n \tau)}, n \tau<t \leq(n+L) \tau, \\
S_{0}-\left[S_{0}-(1-d)\left(S_{0}-\left(S_{0}-S_{1}^{*}\right) \mathrm{e}^{-D L \tau}\right)\right] \mathrm{e}^{-D(t-(n+L) \tau)},(n+L) \tau<t \leq(n+1) \tau,
\end{array}\right. \\
\widetilde{Z_{6}(t)}=\left\{\begin{array}{l}
Z_{6}^{*} \mathrm{e}^{-\left(D+\frac{m m_{1}^{*}}{\delta K}\right)(t-n \tau)}, n \tau<t \leq(n+L) \tau, \\
{\left[Z_{6}^{*} \mathrm{e}^{-\left(D+\frac{m m_{1}^{*}}{\delta K}\right)}+d\left(S_{0}-\left(S_{0}-S_{1}^{*}\right) \mathrm{e}^{-D L \tau}\right)\right] \mathrm{e}^{-D(t-(n+L) \tau)},(n+L) \tau<t \leq(n+1) \tau}
\end{array}\right.
\end{array}\right.
$$

where

$$
Z_{6}^{*}=\frac{B\left[1-(1-d) \mathrm{e}^{-D \tau}\right]+A d \mathrm{e}^{-D \tau}}{\left(1-\mathrm{e}^{-\left(D+\frac{m m_{1}^{*} L}{\delta K}\right) \tau}\right)\left[1-(1-d) \mathrm{e}^{-D \tau}\right]}>0 .
$$

There exists $T_{2} \geq t_{0}+\tau_{1}$, such that

$$
S_{2}(t)>\breve{S}_{2}-\varepsilon_{1}=: \beta \text { for all } t>T_{2},
$$

where $\breve{S}_{2}=Z_{6}^{*}\left(\mathrm{e}^{-\left(D+\frac{m m_{1}^{*}}{\delta K}\right) L \tau}+\mathrm{e}^{-\left(D+\frac{m m_{1}^{*} L}{\delta K}\right) \tau}\right)+d S_{1}^{*} \mathrm{e}^{-D \tau}+d S_{0} \mathrm{e}^{-D \tau}\left(\mathrm{e}^{D L \tau}+1\right)$.

Take $t^{*}=\min \left\{T_{1}, T_{2}\right\}$. For any $t \geq t^{*}$, from (4.3) and (4.9) we obtain

$$
\dot{V}(t)>D\left[\frac{m \mathrm{e}^{-D \tau_{1}} \beta}{D(K+\beta)}-1\right] x(t)
$$

Let

$$
\tilde{x}=\min _{t \in\left[t^{*}, t^{*}+\tau_{1}\right]} x(t) .
$$

In what follows, we prove that $x(t)<\tilde{x}$ for all $t \geq t^{*}$. Otherwise, there exists a positive constant $T_{3}$, for any $t \in\left[t^{*}, t^{*}+\tau_{1}+T_{3}\right]$, we have $x(t) \geq \tilde{x}$, 
$x\left(t^{*}+\tau_{1}+T_{3}\right)=\tilde{x}$ and $x^{\prime}\left(t^{*}+\tau_{1}+T_{3}\right) \leq 0$. Therefore, according to the third equation of (1.2) and (4.10), we further obtain

$$
x^{\prime}\left(t^{*}+\tau_{1}+T_{3}\right)>\left[\frac{m \mathrm{e}^{-D \tau_{1}} \beta}{(K+\beta)}-D\right] x(t)=D\left[\frac{m \mathrm{e}^{-D \tau_{1}} \beta}{D(K+\beta)}-1\right] x(t)>0
$$

which is a contradiction. So we have that $x(t) \geq \tilde{x}>0$ for any $t \geq t^{*}$. From (4.4) we have that $\dot{V}(t)>D\left[\frac{m \mathrm{e}^{-D \tau_{1}} \beta}{D(K+\beta)}-1\right] \tilde{x}>0$ and from which we obtain $V(t) \rightarrow+\infty$ as $t \rightarrow+\infty$. This contradict to $V(t)<\left(1+m \tau_{1} \mathrm{e}^{-D \tau_{1}}\right) M$. Hence, for any nonnegative constant $t_{0}$ with $t \geq t_{0}$, the inequality $x(t)<m_{1}^{*}$ is not hold.

On the one hand, if $x(t)>m_{1}^{*}$ always holds for $t$ large enough, then our target is obtained. Otherwise, suppose $x(t)$ is oscillatory about $m_{1}^{*}$.

Let

$$
m_{1}=\min \left\{\frac{m_{1}^{*}}{2}, m_{1}^{*} \mathrm{e}^{-D \tau_{1}}\right\} .
$$

In what follows, we shall prove that $x(t) \geq m_{1}$ for all $t \geq t_{0}$. There exist two positive integer $\tilde{t}$ and $\omega$ such that $x(\tilde{t})=x(\tilde{t}+\omega)=m_{1}^{*} \quad$ and $\quad x(t)<m_{1}^{*} \quad$ for $\quad \tilde{t}<t<\tilde{t}+\omega$. When $\tilde{t}$ is large enough, $S(t)>\beta$ is hold true for any $\tilde{t}<t<\tilde{t}+\omega$. Since $x(t)$ is uniformly continuous without impacted by pulse. Therefore, for any $T_{3} \in\left(-\tau_{1}, 0\right)$ and $\tilde{t} \leq t \leq \tilde{t}+T_{3}$, we have $x^{\prime}(t)>D\left[\frac{m \mathrm{e}^{-D \tau_{1}} \beta}{D(K+\beta)}-1\right] x(t)$. By this, we have

$$
x(t)>m_{1}^{*} \mathrm{e} \int_{\tilde{t}}^{t}\left(D\left[\frac{m \mathrm{e}^{-D \tau_{1}} \beta}{D(K+\beta)}-1\right]\right) \mathrm{d} v=: \frac{m_{1}^{*}}{2}
$$

for $\tilde{t} \leq t \leq \tilde{t}+T_{3}$. When $\omega \leq T_{3}$, our goal is obtained. When $T_{3}<\omega \leq \tau_{1}$, we have from the third equation of (1.2) that $x^{\prime}(t) \geq-D x(t)$. According to $x(\tilde{t})=m_{1}^{*}$, we obtain $x(t) \geq x(\tilde{t}) \mathrm{e}^{-D \tau_{1}}=m_{1}^{*} \mathrm{e}^{-D \tau_{1}}$ for $\tilde{t}<t \leq \tilde{t}+\omega$. Then $x(t) \geq m_{1}$ can hold true for $\tilde{t}<t \leq \tilde{t}+\omega$. For $\omega \geq \tau_{1}$ and $\tilde{t}<t \leq \tilde{t}+\tau_{1}$, we obtain that $x(t) \geq m_{1}$. By above similar argument, we can show that $x(t) \geq m_{1}$ for $\tilde{t}+\tau_{1} \leq t \leq \tilde{t}+\omega$. Since the interval $[\tilde{t}, \tilde{t}+\omega]$ is arbitrarily chosen by us, we get that $x(t) \geq m_{1}$ for $t$ large enough. In view of our above arguments, the choice of $m_{1}$ is independent of any solution of (1.2) which satisfies that $x(t) \geq m_{1}$ for $t$ large enough. So $x(t) \geq m_{1}$ is hold true for $t$ large enough.

For all $t>0$, from lemma 3 we have that $S_{1}(t) \leq M, S_{2}(t) \leq M, x(t) \leq M$. From Theorem 1, we have $S_{1}(t)>\hat{S}_{1}-\varepsilon_{2}$ and $S_{2}(t)>\hat{S}_{2}-\varepsilon_{2}$ as $t \rightarrow \infty$ and $\varepsilon_{2} \rightarrow 0$, where

$$
\hat{S}_{1}=S_{0}-\left(S_{0}-S_{1}^{*}\right) \mathrm{e}^{-D L \tau}+S_{0}-\left[S_{0}-(1-d)\left(S_{0}-\left(S_{0}-S_{1}^{*}\right) \mathrm{e}^{-D L \tau}\right)\right] \mathrm{e}^{-D(1-L) \tau}
$$

and

$$
\hat{S}_{2}=S_{2}^{*} \mathrm{e}^{-D L \tau}+\left[S_{2}^{*} \mathrm{e}^{-D L \tau}+d\left(S_{0}-\left(S_{0}-S_{1}^{*}\right) \mathrm{e}^{-D L \tau}\right)\right] \mathrm{e}^{-D(1-L) \tau}
$$


So $S_{1}(t)$ and $S_{2}(t)$ are permanent. The proof of Theorem 2 is completed. According to Theorem 1 and 2, we may derive the following conclusion.

Corollary

1) The plankton-extinction periodic solution $\left(\widetilde{S_{1}(t)}, \widetilde{S_{2}(t)}, 0\right)$ is globally attractive if and only if

$$
\tau_{1}>\frac{1}{D} \ln \frac{m \hat{S}_{2}}{D\left(K+\hat{S}_{2}\right)},
$$

where these parametric are the same as the Theorem 1.

2) The plankton $x(t)$ of System (1.2) is permanent if and only if

$$
\tau_{1}<\frac{1}{D} \ln \frac{m \breve{S}_{2}}{D\left(K+\breve{S}_{2}\right)},
$$

where $\breve{S}_{2}=Z_{6}^{*}\left(\mathrm{e}^{-\left(D+\frac{m m_{1}^{*}}{\delta K}\right) L \tau}+\mathrm{e}^{-\left(D+\frac{m m_{1}^{*} L}{\delta K}\right) \tau}\right)+d S_{1}^{*} \mathrm{e}^{-D \tau}+d S_{0} \mathrm{e}^{-D \tau}\left(\mathrm{e}^{D L \tau}+1\right)$.

\section{Discussions and Numerical Analysis}

In this paper, we investigate the necessary and sufficient conditions for the plankton-extinction periodic solution $\left(\widetilde{S_{1}(t)}, \widetilde{S_{2}(t)}, 0\right)$ and permanence of system (1.2). If the time delay $\tau_{1}$ exceeds a certain amount of time, the plankton of system (1.2) will become extinct. If the time delay $\tau_{1}$ is under a certain amount of time, the plankton will be lasting survival in the system. So delay plays an important role in affecting the dynamic behavior of the system. Next, we use numerical simulation to illustrate our mathematical results.

From Theorem 1, we consider dynamical behavior of the system (1.2) with $D=1, \quad S_{0}=2.5, \quad L=0.5, \quad \tau=1, \quad d=0.5, \quad \delta=0.05, \quad \mu_{1}=2.1, \quad \mu_{2}=0.5$, $\tau_{1}=0.5, \quad k=1, \quad m=1, A=3.382, \quad B=0.992, \quad S_{1}^{*}=4.145, \quad S_{2}^{*}=2.773$, $\hat{S}_{2}=3.762$. From (3.7) we obtain that $0.479=\mathrm{e}^{-D \tau_{1}} \frac{m \hat{S}_{2}}{K+\hat{S}_{2}}<D=1$. The plankton-extinction solution $\left(\widetilde{S_{1}(t)}, \widetilde{S_{2}(t)}, 0\right)$ is globally attractive; the plankton of system (1.2) will become extinct in this case.

From Theorem 2, we consider dynamical behavior of the system (1.2) with $D=0.95, \quad S_{0}=4.21, L=0.5, \tau=1, d=0.56, \delta=48, \quad \mu_{1}=1.5, \quad \mu_{2}=3.5$, $\tau_{1}=0.3, \quad k=4.6, \quad m=19.5, \quad A=3.542, \quad B=4.055, \quad \breve{S}_{2}=6.859$, $m_{1}^{*}=13.157$. From (4.1) we obtain that $8.777=\mathrm{e}^{-D \tau_{1}} \frac{m \breve{S}_{2}}{K+\breve{S}_{2}}>D=0.95$. The plankton $x(t)$ of System (1.2) is permanent; the plankton will be lasting survival in the system.

It is difficult to study the global attractivity of system (1.2) analytically. From the numerical simulation (Figure 1) we see that there has a unique $T$-period solution $\left(S_{1}^{*}(t), S_{2}^{*}(t), x^{*}(t)\right)$ of system (1.2) which is globally attractive. The numerical simulation (Figure 2) also shows that system (1.2) is permanent. In 


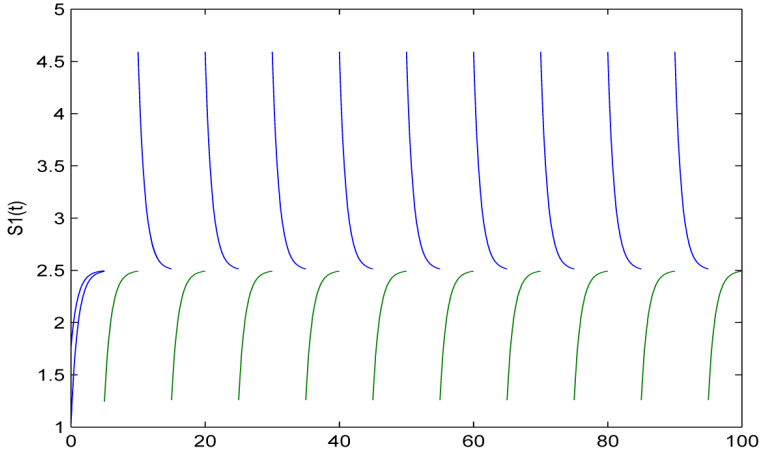

(a)

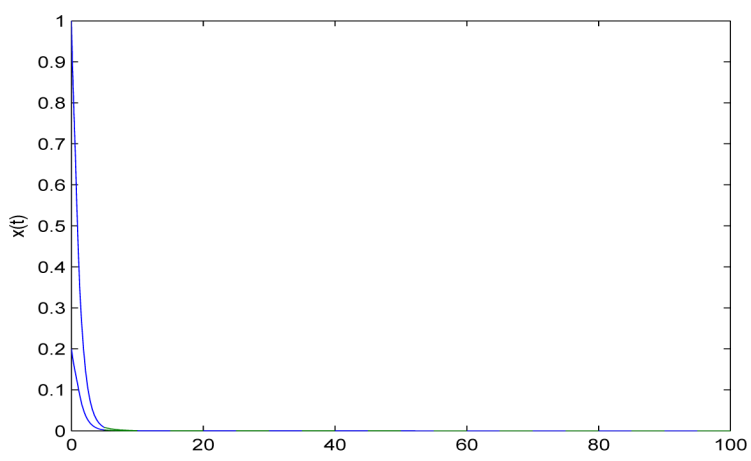

(c)

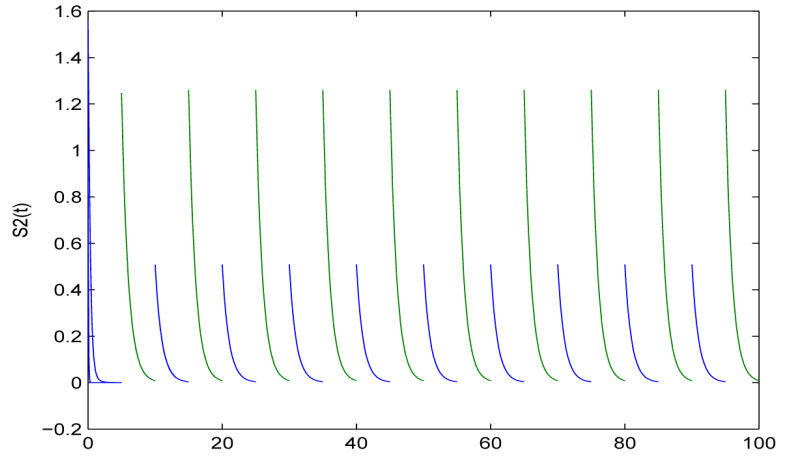

(b)

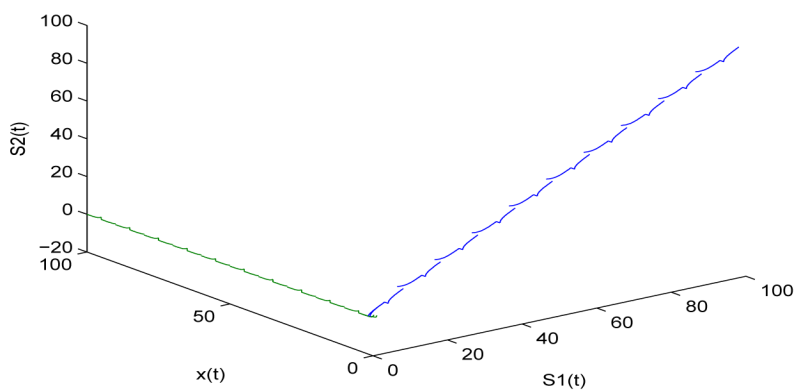

(d)

Figure 1. The plankton-extinction solution of system (1.2) is globally attractive.

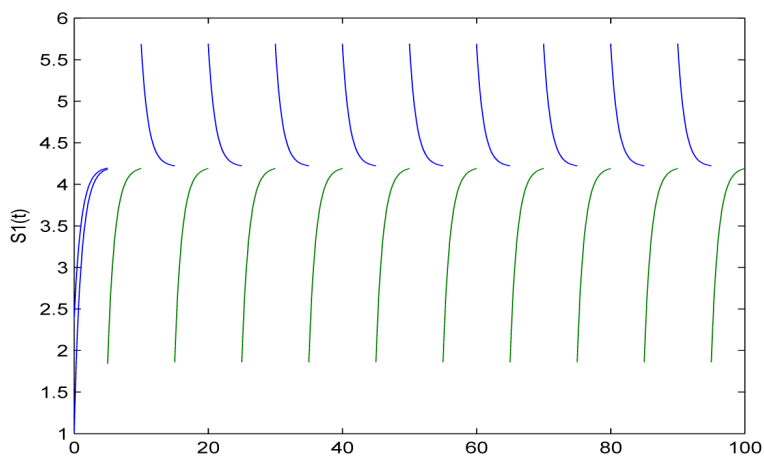

(a)

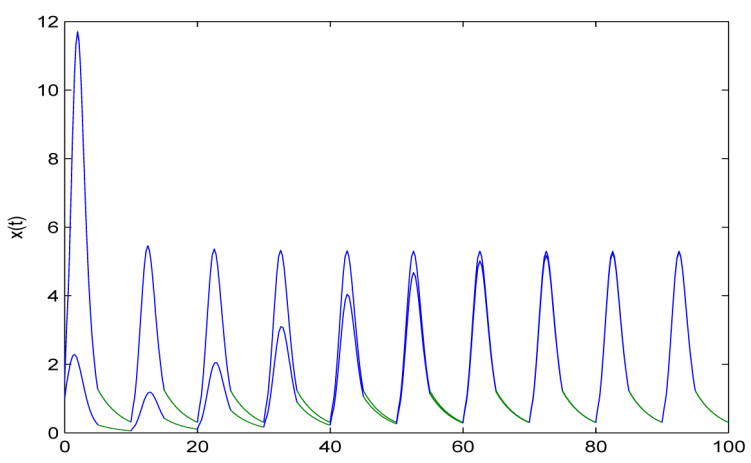

(c)

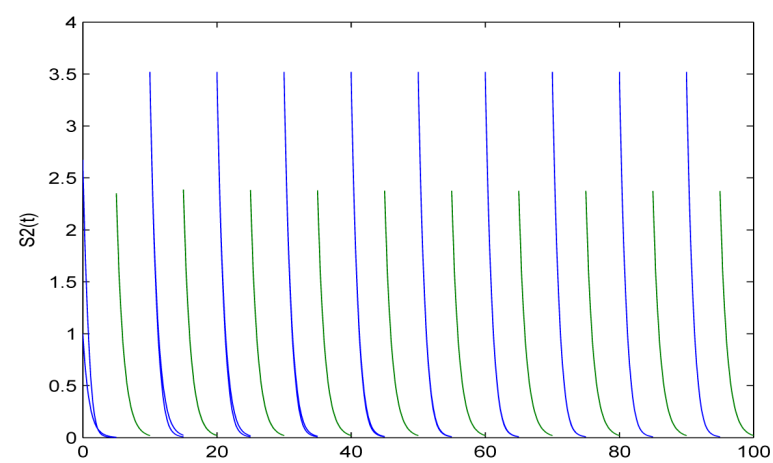

(b)

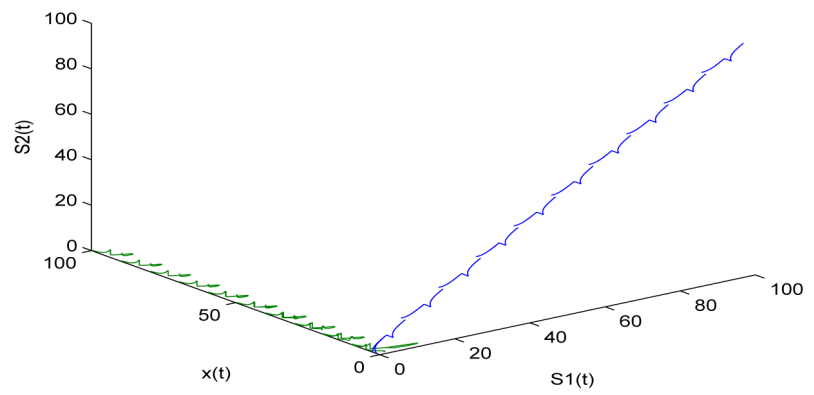

(d)

Figure 2. The plankton $x(t)$ of System (1.2) is permanent. 
view of analytical results, we showed the possibility of establishing control strategy of system (1.2) based on impulsive diffusion and time delay.

\section{References}

[1] Bush, A.W. and Somolinas, A.E. (1975) The Effect of Time Delay and Growth Rate Inhibition in the Bacterial Treatment of Wastewater. Journal of Theoretical Biology, 63, 385-395. https://doi.org/10.1016/0022-5193(76)90041-2

[2] Smith, H. and Waltman, P. (1999) Perturbation of a Globally Stable Ateady State. Proceedings of the AMS, 127, 447-453. https://doi.org/10.1090/S0002-9939-99-04768-1

[3] Hsu, S.B., Waltman, P. and Ellermeyer, S.F. (1994) A Remark on the Global Asymptotic Stability of a Dynamical System Modeling Two Species Competition. Hiroshima Mathematical Journal, 24, 435-445.

[4] Ellermeyer, S., Hendrix, J. and Ghoochan, N. (2003) A Theoretical and Empirical Investigation of Delayed Growth Response in the Continuous Culture of Bacteria. Journal of Theoretical Biology, 222, 485-494. https://doi.org/10.1016/S0022-5193(03)00063-8

[5] Bulert, G.L., Hsu, S.B. and Waltman, P. (1985) A Mathematical Model of the Chemostat with Periiodic Washout Rate. SIAM Journal on Applied Mathematics, 45, 435-449. https://doi.org/10.1137/0145025

[6] Bainov, D. and Simeonov, P. (1993) Impulsive Differential Equations: Periodic Solutions and Applications. Longman, Harlow.

[7] Jiao, J. and Chen, L. (2008) Global Attractivity of a Stage-Structure Variable Coefficients Predator-Prey System with Time Delay and Impulsive Perturbations on Predators. International Journal of Biomathematics, I, 197-208. https://doi.org/10.1142/S1793524508000163

[8] Smith, R.J. and Wolkowicz, G.S.K. (2004) Analysis of a Model of the Nutrient Driven Self-Cycling Fermentation Process. Dynamics of Continuous, Discrete and Impulsive Systems Series B, 11, 239-265.

[9] Wang, L.M., Chen, L.S., et al. (2009) Impulsive Diffusion in Single Species Model. Chaos, Solitons \& Fractals, 33, 1213-1219. https://doi.org/10.1016/j.chaos.2006.01.102

[10] Wang, L.C.H. and Lee, T.F. (1996) Torpor and Hibernation in Manmals: Metabolic, Physiological and Chemical Adaptations. In: Fregley, M.J., Blatteis, C.M., Eds., Handbook of Physiology: Environmental Physiology, Oxford University Press, New York, 507-532.

[11] Staples, J.F. and Brown, J.C.L. (2008) Mitochondrial Metabolism in Hibernation and daily Torpor: A Review. Journal of Comparative Physiology B, 178, 811-827. https://doi.org/10.1007/s00360-008-0282-8

[12] Levin, S.A. and Segel, L.A. (1976) An Hypothesis for the Origin of Planktonic Patchiness. Nature, 259, 659. https://doi.org/10.1038/259659a0

[13] Okubo, A. (1980) Diffusion and Ecological Problems: Mathematical Models. Springer-Verlag, Berlin.

[14] Ruan, S. (1998) Turing Instability and Traveling Waves in Diffusive Plankton Models with Delayed Nutrient Recyling. IMA Journal of Applied Mathematics, 61, 1532. https://doi.org/10.1093/imamat/61.1.15

[15] Jiao, J., Chen, L. and Cai, S. (2016) Dynamics of a Plankton-Nutrient Chemostat Model with Hibernation and It Described by Impulsive Switched Systems. Journal of Applied Mathematics and Computing, 35, 211-227. 
https://doi.org/10.1007/s12190-015-0983-6

[16] Zhang, T., Ma, W. and Meng, X. (2015) Dynamical Analysis of a Continuous-Culture and Harvest Chemostat Model with Impulsive Effect. Journal of Biological Sys tems, 23, Article ID: 1550028, 21 p.

[17] Jiao, J. and Chen, L. (2009) Dynamical Analysis of a Chemostat Model with Delayed Response in Growth and Pulse Input in Polluted Environment. Journal of Mathematical Chemistry, 46, 502-513. https://doi.org/10.1007/s10910-008-9474-4

Submit or recommend next manuscript to SCIRP and we will provide best service for you:

Accepting pre-submission inquiries through Email, Facebook, LinkedIn, Twitter, etc. A wide selection of journals (inclusive of 9 subjects, more than 200 journals)

Providing 24-hour high-quality service

User-friendly online submission system

Fair and swift peer-review system

Efficient typesetting and proofreading procedure

Display of the result of downloads and visits, as well as the number of cited articles

Maximum dissemination of your research work

Submit your manuscript at: http://papersubmission.scirp.org/

Or contact jamp@scirp.org 